\title{
Article \\ Multiple Scattering by Two PEC Spheres Using Translation Addition Theorem
}

\author{
Sidra Batool ${ }^{1}$ *(D), Mehwish Nisar ${ }^{1}$, Lorenzo Dinia ${ }^{1}$, Fabio Mangini ${ }^{2} \mathbb{D}$ and Fabrizio Frezza ${ }^{1}$ (D) \\ 1 Department of Information Engineering, Electronics, and Telecommunications, Sapienza University of Rome, \\ Via Eudossiana 18, 00184 Rome, Italy; mehwish.nisar@uniroma1.it (M.N.); lorenzo.dinia@uniroma1.it (L.D.); \\ fabrizio.frezza@uniroma1.it (F.F.) \\ 2 Department of Information Engineering, University of Brescia, Via Branze 59, 25123 Brescia, Italy; \\ fabio.mangini@unibs.it \\ * Correspondence: sidra.batool@uniroma1.it
}

Citation: Batool, S.; Nisar, M.; Dinia,

L.; Mangini, F.; Frezza, F. Multiple

Scattering by Two PEC Spheres Using Translation Addition Theorem.

Electronics 2022, 11, 126. https://

doi.org/10.3390/electronics11010126

Academic Editor: Gerardo Di Martino

Received: 18 November 2021

Accepted: 25 December 2021

Published: 31 December 2021

Publisher's Note: MDPI stays neutral with regard to jurisdictional claims in published maps and institutional affiliations.

Copyright: (c) 2021 by the authors. Licensee MDPI, Basel, Switzerland. This article is an open access article distributed under the terms and conditions of the Creative Commons Attribution (CC BY) license (https:// creativecommons.org/licenses/by/ $4.0 /)$.

\begin{abstract}
An analysis of multiple scattering by two Perfect Electric Conducting (PEC) spheres using translation Addition Theorem (AT) for spherical vector wave functions is presented. Specifically, the Cruzan formalism is used to represent the AT for spherical harmonics, which introduces the translation coefficients for transformation of spherical harmonics from one coordinate to another. The adoption of these coefficients with the use of two PEC spheres in a near zone region makes the calculation of multiple scattering electric fields very efficient. As an illustration, the mathematical formation using advanced computational approaches was inspected. Then, the generic truncation criteria in the scattered electric field by two PEC spheres was deeply investigated using translation AT. However, the numerical validation was obtained using Comsol simulation software. This approach will allow to evaluate the scattering from macro-structures composed of spherical particles, i.e., biological molecules, clouds of airborne particles, etc. An original and fully general solution to the problem using vector quantities is introduced, and the convergence of the solution in several numerical examples is also demonstrated. This approach takes into account the effect of multiple scattering by two PEC spheres for spherical vector function.
\end{abstract}

Keywords: scattering; translation addition theorem; Mie theory

\section{Introduction}

The problem of multiple scattering by closely spaced objects has a wide range of engineering applications, including electromagnetic (EM) wave transmission by rain [1], scattering by complex bodies [2-5], scanning of buried objects [6,7], biological cell detection [8,9], radar and remote sensing applications in biomedical diagnostics, etc. $[10,11]$. The resolution of multiple scattering problems by PEC spheres allows an analytic treatment and a better physical interpretation into the scattering mechanism for novel applications [12,13].

The problem of scattering from two identical spheres with small radii was formulated by Liang and Lo [14]. The translation AT for vector spherical wave functions as multipole expansion was used to express the derived solution of EM fields distributed by spheres. Later on, Olaofe [15] described the multiple scattering by an unequal and parallel circular cylinders. An overview of previous research projects on multiple scattering problems showed that a theoretical investigation of the effects of inter-particle coupling on morphology-dependent resonances of spheres was examined by Fuller [16]. Lo and Bruning determined the new recursion relationship for the calculations of the multiple scattering of EM waves by two arbitrary spheres, reducing the difficulty of the computational quantitative analysis in scattering problems [17]. Further, Wang and Chew derived the recursive approach (T-matrix algorithm), which is used for the formation of multiple scattering fields by several spheres. This method is suitable for the calculation of the vector AT and valid for Monte Carlo simulation for many diagnostic applications [18]. 
In 1954, Friedman and Russek proposed the calculation of AT for spherical scalar wave functions [19]. Cruzen and Stein also developed the AT for spherical harmonics, fitting it for the appropriate solution of multiple scattering by spheres [20,21]. More recently, a further improvement was introduced by Mackowski with the superposition solution method for multiple spheres scattering problems [22]. Xu illustrated the multiple scattering by aggregate spheres, and interpreted the detail of the fast evaluation of Gaunt coefficients [23]. The vector translation AT for spherical harmonics was first formulated by $\mathrm{Xu}$ for an analytical solution of multiple scattering problems. Then, the computational difference between the three vital types of analytical expressions was determined for the vector translation coefficients: Stein's [20], Cruzan's [21], and Xu's [24]. Moreover, Xu presented the necessary recursive approaches to exactly calculating the Gaunt coefficients using Wigner 3-j [25].

Recently, Batool et al. specified a brief outline of the multiple scattering by a PEC sphere using translation AT for spherical harmonics [26]. Additionally, the effect of truncation error on its convergence behavior in AT for spherical harmonics was deduced [27]. As is known, some researchers reported the truncation error and its convergence in Mie theory [28]. It was observed that the most popular Wiscombe's criterion was not sufficient to overcome the truncation error of translation AT for spherical vector functions [29].

In the current manuscript, the multiple scattering analysis using two PEC spheres was studied. The Cruzen formula for the translation addition coefficients based on Wigner 3-j was selected. Then, the scattering electric field using AT for spherical harmonics was derived and the mathematical formation using numerical simulation approach was computed. During our numerical investigation, the truncation error and its convergence were observed to vary with respect to the frequency. The study offers an examination of the impact of finite terms on the truncation error of AT related to spherical vector wave function. The truncation effect forces the numerical results of spherical harmonics to vary, as common for the most truncation criteria used. Currently, the most used truncation criterion in the literature is the one proposed by Wiscombe more than 40 years ago. This criterion allows to accurately choose the truncation of the series in order to express an electric field in the presence of a single scatterer. On the other hand, it does not take into consideration more complex scenarios, as in the case of two or more scatterers. In this scenario, the field must be expressed in spherical vector wave functions translated on the center of a reference system of each other sphere. This translation is reached by exploiting the AT, which in turn is obtained by a superposition of vector spherical functions that must obey an appropriate truncation criterion. As a consequence of the numerical simulation results, the translation of both spheres along $z$-axis and the observed scattering pattern by varying frequency and radii of the spheres were presented. Comsol (Multiphysics 5.4) simulation software for the validation of the numerical results was used and the best comparison between Matlab and Comsol results was also retrieved.

\section{Formation of the Problem}

Two PEC spheres with radii $a$ and $c$ with respect to the center at the origins $O$ and $O^{\prime}$ of two different coordinates systems are taken under consideration. The sphere with $O$ as origin is characterized by a spherical coordinates system $(r, \theta, \phi)$, while $\left(r^{\prime}, \theta^{\prime}, \phi^{\prime}\right)$ characterizes the sphere with origin $O^{\prime}$. The distance between the centers of spheres along $z$-axis is $\delta$ as shown in Figure 1. Let us study a horizontal elliptically polarized plane wave that propagates along a certain direction of the three dimensions cartesian coordinates system. The plane wave can be written as [30,31]:

$$
\mathbf{E}_{i}(\mathbf{r})=\mathbf{e}_{\text {pol }} \exp \left(i \mathbf{k}_{i} \mathbf{r}\right)=\left(E_{\theta} \boldsymbol{\theta}+E_{\phi} \boldsymbol{\phi}\right) \exp \left(i \mathbf{k}_{i} \mathbf{r}\right)
$$


with

$$
\begin{aligned}
\mathbf{k}_{i} & =k_{1}\left(\sin \theta_{i} \cos \phi_{i} \mathbf{x}_{\mathbf{0}}+\sin \theta_{i} \sin \phi_{i} \mathbf{y}_{\mathbf{0}}+\cos \theta_{i} \mathbf{z}_{\mathbf{0}}\right) \\
\boldsymbol{\theta}_{0 i} & =\left(\cos \theta_{i} \cos \phi_{i} \mathbf{x}_{\mathbf{0}}+\cos \theta_{i} \sin \phi_{i} \mathbf{y}_{\mathbf{0}}-\sin \theta_{i} \mathbf{z}_{\mathbf{0}}\right) \\
\boldsymbol{\phi}_{0 i} & =-\sin \phi_{i} \mathbf{x}_{\mathbf{0}}+\cos \phi_{i} \mathbf{y}_{\mathbf{0}}
\end{aligned}
$$

Then, the incident electric field in terms of spherical harmonics can be written as [30,31]:

$$
\mathbf{E}_{i}(\mathbf{r})=\sum_{\nu=1}^{+\infty} \sum_{\mu=-v}^{v}\left[a_{\mu \nu} \mathbf{M}_{\mu \nu}^{(1)}(\mathbf{r})+b_{\mu \nu} \mathbf{N}_{\mu \nu}^{(1)}(\mathbf{r})\right]
$$

Vector spherical harmonics $\mathbf{M}_{\mu \nu}^{(1)}$ and $\mathbf{N}_{\mu \nu}^{(1)}$ is $[30,31]$ :

$$
\begin{aligned}
\mathbf{M}_{\mu \nu}^{(1)} & =\exp (i \mu \phi) j_{v}(k r)\left[i \mu \frac{P_{v}^{\mu}(\cos \theta)}{\sin \theta} \boldsymbol{\theta}_{0}-\frac{\partial P_{v}^{\mu}(\cos \theta)}{\partial \theta} \boldsymbol{\phi}_{0}\right] \\
\mathbf{N}_{\mu \nu}^{(1)} & =\exp (i \mu \phi) \frac{j_{v}(k r)}{k r} v(v+1) P_{v}^{\mu}(\cos \theta) \boldsymbol{r}_{0}+ \\
& {\left[\frac{\partial P_{v}^{\mu}(\cos \theta)}{\partial \theta} \boldsymbol{\theta}_{0}+i \frac{\mu P_{v}^{\mu}(\cos \theta)}{\sin \theta} \boldsymbol{\phi}_{0}\right] \exp (i \mu \phi) \frac{1}{k r} \frac{\partial}{\partial r}\left[r j_{v}(k r)\right] }
\end{aligned}
$$

considering

$$
\begin{aligned}
\pi_{v}^{\mu}(\cos \theta) & =\mu \frac{P_{v}^{\mu}(\cos \theta)}{\sin \theta} \\
\tau_{v}^{\mu}(\cos \theta) & =\frac{\partial P_{v}^{\mu}(\cos \theta)}{\partial \theta}
\end{aligned}
$$

The vector spherical harmonics expressions can be simplified as follows:

$$
\begin{aligned}
\mathbf{M}_{\mu \nu}^{(1)} & =\exp (i \mu \phi) j_{v}(k r)\left[i \pi_{v}^{\mu}(\cos \theta) \boldsymbol{\theta}_{0}-\tau_{v}^{\mu}(\cos \phi) \boldsymbol{\phi}_{0}\right] \\
\mathbf{N}_{\mu \nu}^{(1)} & =\exp (i \mu \phi) \frac{j_{v}(k r)}{k r} v(v+1) P_{v}^{\mu}(\cos \theta) \mathbf{r}_{0}+ \\
& {\left[\tau_{v}^{\mu}(\cos \theta) \boldsymbol{\theta}_{0}+i \pi_{v}^{\mu}(\cos \phi) \boldsymbol{\phi}_{0}\right] \exp (i \mu \phi) \frac{1}{k r} \frac{\partial}{\partial r}\left[r j_{v}(k r)\right] }
\end{aligned}
$$

Now, let us consider modified spherical vector functions characterized by an angles $\theta$ and $\phi$. The following expressions are obtained replacing the spherical vector function with the tesseral function:

$$
\begin{aligned}
\mathbf{m}_{\mu v} & =\exp (i \mu \phi)\left[i \pi_{v}^{\mu}(\cos \theta) \boldsymbol{\theta}_{0}-\tau_{v}^{\mu}(\cos \theta) \boldsymbol{\phi}_{0}\right] \\
\mathbf{n}_{\mu v} & =\exp (i \mu \phi)\left[\tau_{v}^{\mu}(\cos \theta) \boldsymbol{\theta}_{0}+i \pi_{v}^{\mu}(\cos \theta) \boldsymbol{\phi}_{0}\right] \\
\mathbf{p}_{\mu v} & =\exp (i \mu \phi) v(v+1) P_{v}^{\mu}(\cos \theta) \boldsymbol{r}_{0}
\end{aligned}
$$

By above-simplified Equations (10) and (11), we achieve:

$$
\begin{aligned}
\mathbf{M}_{\mu \nu}^{(1)} & =j_{v}(k r) \mathbf{m}_{\mu v} \\
\mathbf{N}_{\mu \nu}^{(1)} & =\frac{j_{v}(k r)}{k r} \mathbf{p}_{\mu \nu}+\frac{1}{k r} \frac{\partial}{\partial r}\left[r j_{v}(k r)\right] \mathbf{n}_{\mu v}
\end{aligned}
$$


The features of orthogonality properties are used for vector spherical harmonics, which implies that the coefficients can be expressed in a modified form:

$$
\begin{aligned}
& a_{\mu v}=i^{v} \frac{(2 v+1)(v-\mu) !}{v(v+1)(v+\mu) !} \mathbf{e}_{p o l} \cdot \mathbf{m}_{\mu v}^{*}\left(\theta_{t}, \phi_{t}\right) \\
& b_{\mu v}=i^{v-1} \frac{(2 v+1)(v-\mu) !}{v(v+1)(v+\mu) !} \mathbf{e}_{p o l} \cdot \mathbf{n}_{\mu v}^{*}\left(\theta_{t}, \phi_{t}\right)
\end{aligned}
$$

The elliptically polarized incident field for a sphere may once be written as:

$$
\mathbf{E}_{i}(r, \theta, \phi)=\sum_{\nu=1}^{+\infty} \sum_{\mu=-v}^{v}\left[a_{\mu \nu} \mathbf{M}_{\mu \nu}^{(1)}(r, \theta, \phi)+b_{\mu \nu} \mathbf{N}_{\mu \nu}^{(1)}(r, \theta, \phi)\right]
$$

with

$$
\begin{aligned}
& a_{\mu v}=i^{v} \frac{(2 v+1)(v-\mu) !}{v(v+1)(v+\mu)} \exp \left(-i \mu \phi_{i}\right)\left[-i E_{\theta_{i}} \pi_{v}^{\mu}\left(\cos \theta_{i}\right)-E_{\phi_{i}} \tau_{v}^{\mu}\left(\cos \theta_{i}\right)\right] \\
& b_{\mu v}=i^{v-1} \frac{(2 v+1)(v-\mu) !}{v(v+1)(v+\mu)} \exp \left(-i \mu \phi_{i}\right)\left[-i E_{\theta_{i}} \tau_{v}^{\mu}\left(\cos \theta_{i}\right)-E_{\phi_{i}} \pi_{v}^{\mu}\left(\cos \theta_{i}\right)\right]
\end{aligned}
$$

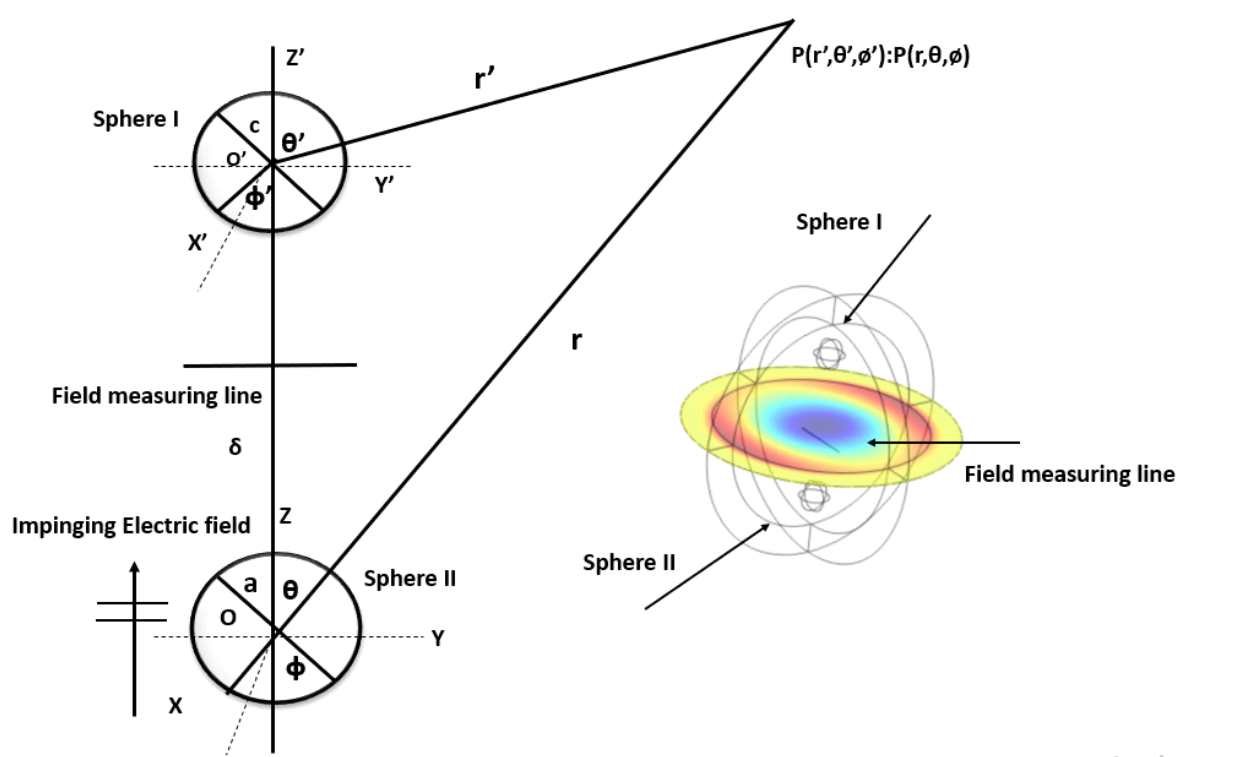

Figure 1. Geometry of the two PEC spheres exercising translation of the spheres along-z-axis using translation AT.

\subsection{Expansion of Incident Plane Wave}

The incident electric field is expanded into multipole fields series around the origins $O$ and $O^{\prime}$ of the spherical coordinates systems $(r, \theta, \phi)$ and $\left(r^{\prime}, \theta^{\prime}, \phi^{\prime}\right)$. Thus, the incident plane wave using multipole coefficients expansion may be written as follows, since the radial vector $r=r^{\prime}+\delta=\exp \left(-i k_{1} r^{\prime}\right) \exp \left(-i k_{1} \delta \cos \alpha\right)$ [14].

$$
\mathbf{E}_{i}\left(r^{\prime}, \theta^{\prime}, \phi^{\prime}\right)=\sum_{v=1}^{+\infty} \sum_{\mu=-v}^{v}\left[a_{\mu v}^{*} \mathbf{M}_{\mu \nu}^{(1)}\left(r^{\prime}, \theta^{\prime}, \phi^{\prime}\right)+b_{\mu v}^{*} \mathbf{N}_{\mu \nu}^{(1)}\left(r^{\prime}, \theta^{\prime}, \phi^{\prime}\right)\right]
$$

where

$$
\begin{aligned}
& a_{\mu v}^{*}=\exp \left(-i k_{1} \delta \cos \alpha\right) a_{\mu v} \\
& b_{\mu v}^{*}=\exp \left(-i k_{1} \delta \cos \alpha\right) b_{\mu v}
\end{aligned}
$$




\subsection{Scattered Field by Two Conducting Sphere}

Scattered field by two PEC spheres may be represented with $\mathbf{E}_{s}^{I}$ and $\mathbf{E}_{s}^{I I}$, respectively. In this problem, the total electric field is described by the incident and scattered electric fields, and it is expressed as follows:

$$
\mathbf{E}^{\text {total }}=\mathbf{E}_{i}+\mathbf{E}_{s}^{I}+\mathbf{E}_{s}^{I I}
$$

where

$$
\begin{aligned}
\mathbf{E}_{s}^{I}(r, \theta, \phi) & =\sum_{\nu=1}^{+\infty} \sum_{\mu=-\nu}^{v}\left[e_{\mu \nu} \mathbf{M}_{\mu \nu}^{(3)}(r, \theta, \phi)+f_{\mu v} \mathbf{N}_{\mu \nu}^{(3)}(r, \theta, \phi)\right] \\
\mathbf{E}_{s}^{I I}\left(r^{\prime}, \theta^{\prime}, \phi^{\prime}\right) & =\sum_{\nu=1}^{+\infty} \sum_{\mu=-v}^{v}\left[g_{\mu v} \mathbf{M}_{\mu \nu}^{(3)}\left(r^{\prime}, \theta^{\prime}, \phi^{\prime}\right)+h_{\mu \nu} \mathbf{N}_{\mu \nu}^{(3)}\left(r^{\prime}, \theta^{\prime}, \phi^{\prime}\right)\right]
\end{aligned}
$$

\subsection{AT for Translation of the Vector Spherical Harmonics}

The translation AT for spherical vector wave equations depends on the relative schism in a spherical coordinates system using different origin $O$ and $O^{\prime}$. The following expressions are used to express the translation of $l_{t h}$ coordinates system $(r, \theta, \phi)$ to $j_{t h}\left(r^{\prime}, \theta^{\prime}, \phi^{\prime}\right)$ coordinates system.

$$
\begin{aligned}
\mathbf{M}_{\mu \nu}^{(3)}(l) & =\sum_{n=1}^{\infty} \sum_{m=-n}^{n} A_{m n}^{\mu \nu}(l, j) \mathbf{M}_{m n}^{(1)}(j)+B_{m n}^{\mu \nu}(l, j) \mathbf{N}_{m n}^{(1)}(j) \\
\mathbf{N}_{\mu v}^{(3)}(l) & =\sum_{n=1}^{\infty} \sum_{m=-n}^{n} B_{m n}^{\mu v}(l, j) \mathbf{M}_{m n}^{(1)}(j)+A_{m n}^{\mu \nu}(l, j) \mathbf{N}_{m n}^{(1)}(j)
\end{aligned}
$$

Then, translation of a $j$ th coordinates system $\left(r^{\prime}, \theta^{\prime}, \phi^{\prime}\right)$ to $l$ th coordinates system $(r, \theta, \phi)$ at translation distance $\delta$ may be formulated as:

$$
\begin{aligned}
\mathbf{M}_{\mu \nu}^{(3)}(j) & =\sum_{n=1}^{\infty} \sum_{m=-n}^{n} C_{m n}^{\mu v}(j, l) \mathbf{M}_{m n}^{(1)}(l)+D_{m n}^{\mu v}(j, l) \mathbf{N}_{m n}^{(1)}(l) \\
\mathbf{N}_{\mu \nu}^{(3)}(j) & =\sum_{n=1}^{\infty} \sum_{m=-n}^{n} D_{m n}^{\mu v}(j, l) \mathbf{M}_{m n}^{(1)}(l)+C_{m n}^{\mu v}(j, l) \mathbf{N}_{m n}^{(1)}(l)
\end{aligned}
$$

\subsection{Formation of Vector Translation Coefficients}

EM field in terms of translation is illustrated by an infinite sum with respect to a coordinate system with a different reference. Therefore, Cruzan's mathematical derivations for $A_{m n \mu \nu}^{l, j}$ and $B_{m n \mu \nu}^{l, j}$ coefficients can be written in a simplified form [21]:

$$
\begin{array}{r}
A_{m n \mu v}^{l, j}=(-1)^{-m} \frac{(2 v+1)(n+m) !(v-\mu) !}{2 n(n+1)(n-m) !(v+\mu) !} \exp \left[i(\mu-m) \phi_{l j}\right] \\
\times \sum_{q=0}^{q_{\max }} i^{p}[n(n+1)+v(v+1)-p(p+1)] a_{q} \\
\times h_{p}^{(1)}(k d) P_{p}^{\mu-m}(\cos \theta)
\end{array}
$$

The following expressions are reached:

$$
\begin{aligned}
& A_{m n \mu \nu}^{l, j}=(-1)^{-m} \zeta(m, n, \mu, v) \sum_{q=0}^{q_{\max }} h_{p}^{(1)}(k r) \\
& C_{m n \mu v}^{l, j}=(-1)^{-m} \zeta^{*}(m, n, \mu, v) \sum_{q=0}^{q_{\max }} h_{p}^{(1)}(k r)
\end{aligned}
$$


where

$$
\begin{aligned}
& \zeta(m, n, \mu, v)=\frac{(2 v+1)(n+m) !(v-\mu) !}{2 n(n+1)(n-m) !(v+\mu) !} \exp \left[i(\mu-m) \phi_{l j}\right] \\
& \times \sum_{q=0}^{q_{\max }} i^{p}[n(n+1)+v(v+1)-p(p+1)] a_{q} \quad P_{p}^{\mu-m}\left(\cos \theta_{l j}\right)
\end{aligned}
$$

and

$$
\begin{aligned}
& B_{m n \mu v}^{l, j}=(-1)^{-m+1} \frac{(2 v+1)(n+m) !(v-\mu) !}{2 n(n+1)(n-m) !(v+\mu) !} \exp \left[i(\mu-m) \phi_{l j}\right] \\
\times & \sum_{q=1}^{Q_{\max }} i^{p+1}\left\{\left[(p+1)^{2}-(n-v)^{2}\right]\left[(n+v+1)^{2}-(p+1)^{2}\right]\right\}^{\frac{1}{2}} \\
\times & b(-m, n, \mu, v, p+1, p) h_{p+1}^{(1)}(k r) P_{p+1}^{\mu-m}(\cos \theta)
\end{aligned}
$$

Similarly

$$
\begin{aligned}
& B_{m n \mu \nu}^{l, j}=(-1)^{-m+1} \xi(m, n, \mu, v) \sum_{q=0}^{q_{\max }} h_{p+1}^{(1)}(k r) \\
& D_{m n \mu v}^{l, j}=(-1)^{-m+1} \xi^{*}(m, n, \mu, v) \sum_{q=0}^{q_{\max }} h_{p+1}^{(1)}(k r)
\end{aligned}
$$

where

$$
\begin{aligned}
& \xi(m, n, \mu, v)=\frac{(2 v+1)(n+m) !(v-\mu) !}{2 n(n+1)(n-m) !(v+\mu) !} \exp \left[i(\mu-m) \phi_{l j}\right] \\
\times & \sum_{q=1}^{Q_{\max }} i^{p+1}\left\{\left[(p+1)^{2}-(n-v)^{2}\right]\left[(n+v+1)^{2}-(p+1)^{2}\right]\right\}^{\frac{1}{2}} \\
& \times b(-m, n, \mu, v, p+1, p) P_{p+1}^{\mu-m}\left(\cos \theta_{l j}\right)
\end{aligned}
$$

Similarly, $C_{m n \mu v}^{j, l}$ and $D_{m n \mu v}^{j, l}$ coefficients are obtained by taking the complex conjugate of $\xi(m, n, \mu, v)$ and $\zeta(m, n, \mu, v)$ from $A_{m n \mu v}^{j, l}$ and $B_{m n \mu \nu}^{j, l}$. Here, $k$ is the propagation constant, $a_{q}=a(-m, n, \mu, v, p), q=1,2, \ldots, q_{\max }, p=n+v-2 q$ and

$$
q_{\max }=\min \left(n, v, \frac{n+v-|m+\mu|}{2}\right)
$$

Gaunt coefficients have been described in the literature [24], the total electric field for the $l$ th coordinates system $(r, \theta, \phi)$ may be explicitly written as:

$$
\begin{aligned}
\mathbf{E}_{\text {total }}(l) & =\sum_{v=1}^{+\infty} \sum_{\mu=-v}^{v} a_{\mu v} \mathbf{M}_{\mu v}^{(1)}(l)+b_{\mu v} \mathbf{N}_{\mu \nu}^{(1)}(l)+e_{\mu v} \mathbf{M}_{\mu \nu}^{(3)}(l)+f_{\mu v} \mathbf{N}_{\mu v}^{(3)}(l) \\
& +\sum_{v=1}^{+\infty} \sum_{\mu=-v}^{v} \sum_{n=1}^{\infty} \sum_{m=-n}^{n} g_{\mu v}\left\{A_{m n}^{\mu v}(l, j) \mathbf{M}_{m n}^{(1)}(l)+B_{m n}^{\mu v}(l, j) \mathbf{N}_{m n}^{(1)}(l)\right\}+ \\
& h_{\mu v}\left\{B_{m n}^{\mu v}(l, j) \mathbf{M}_{m n}^{(1)}(l)+A_{m n}^{\mu v}(l, j) \mathbf{N}_{m n}^{(1)}(l)\right\}
\end{aligned}
$$


Similarly, the total electric field for the $j_{t h}$ coordinates system $\left(r^{\prime}, \theta^{\prime}, \phi^{\prime}\right)$ may be written as:

$$
\begin{aligned}
\mathbf{E}_{\text {total }}(j) & =\sum_{v=1}^{+\infty} \sum_{\mu=-v}^{v} a_{\mu \nu}^{*} \mathbf{M}_{\mu \nu}^{(1)}(j)+b_{\mu \nu}^{*} \mathbf{N}_{\mu \nu}^{(1)}(j)+g_{\mu \nu} \mathbf{M}_{\mu \nu}^{(3)}(j)+h_{\mu v} \mathbf{N}_{\mu \nu}^{(3)}(j) \\
& +\sum_{v=1}^{+\infty} \sum_{\mu=-v}^{v} \sum_{n=1}^{\infty} \sum_{m=-n}^{n} e_{\mu \nu}\left\{C_{m n}^{\mu v}(j, l) \mathbf{M}_{m n}^{(1)}(j)+D_{m n}^{\mu v}(j, l) \mathbf{N}_{m n}^{(1)}(j)\right\}+ \\
& f_{\mu v}\left\{D_{m n}^{\mu v}(j, l) \mathbf{M}_{m n}^{(1)}(j)+C_{m n}^{\mu v}(j, l) \mathbf{N}_{m n}^{(1)}(j)\right\}
\end{aligned}
$$

Applying the boundary condition, the tangential component of the electric field must continue on the surface of spheres. Using the orthogonality properties, the simultaneous linear equations can be expressed as:

$$
\begin{aligned}
& e_{\mu v}=X_{n}(a)\left\{a_{\mu v}+\left(g_{\mu v} \sum_{n=1}^{\infty} \sum_{m=-n}^{n} A_{m n}^{\mu v}+h_{\mu v} \sum_{n=1}^{\infty} \sum_{m=-n}^{n} B_{m n}^{\mu v}\right)\right\} \\
& f_{\mu v}=Y_{n}(a)\left\{b_{\mu v}+\left(g_{\mu v} \sum_{n=1}^{\infty} \sum_{m=-n}^{n} B_{m n}^{\mu v}+h_{\mu \nu} \sum_{n=1}^{\infty} \sum_{m=-n}^{n} A_{m n}^{\mu v}\right)\right\} \\
& g_{\mu \nu}=X_{n}(c)\left\{a_{\mu \nu}^{*}+\left(e_{\mu v} \sum_{n=1}^{\infty} \sum_{m=-n}^{n} C_{m n}^{\mu v}+f_{\mu \nu} \sum_{n=1}^{\infty} \sum_{m=-n}^{n} D_{m n}^{\mu \nu}\right)\right\} \\
& h_{\mu v}=Y_{n}(c)\left\{b_{\mu v}^{*}+\left(e_{\mu v} \sum_{n=1}^{\infty} \sum_{m=-n}^{n} D_{m n}^{\mu v}+f_{\mu v} \sum_{n=1}^{\infty} \sum_{m=-n}^{n} C_{m n}^{\mu v}\right)\right\}
\end{aligned}
$$

where

$$
\begin{aligned}
& X\left(r_{0}\right)=-\left.\frac{j_{v}(k r)}{h_{v}^{(1)}(k r)}\right|_{r=r_{0}} \\
& Y\left(r_{0}\right)=-\left.\frac{j_{v}^{\prime}(k r)}{h_{v}^{\prime(1)}(k r)}\right|_{r=r_{0}}
\end{aligned}
$$

After solving the linear equations, all coefficients $e_{\mu v}, f_{\mu v}, g_{\mu \nu}, h_{\mu \nu}$ can be obtained, thanks to which it is easily possible to determine scattering, extinction, and absorption cross-section [32]. As a result of the above derivations and the use of Equations (28) and (29), the scattered field can be achieved:

$$
\begin{array}{r}
\mathbf{E}_{s}(l)=\sum_{v=1}^{\infty} \sum_{\mu=-v}^{v}\left[e_{\mu v} \sum_{n=1}^{\infty} \sum_{m=-n}^{n}\left\{A_{m n}^{\mu v}(l, j) \mathbf{M}_{m n}^{(3)}(j)+B_{m n}^{\mu v}(l, j) \mathbf{N}_{m n}^{(3)}(j)\right\}\right. \\
\left.+f_{\mu \nu} \sum_{n=1}^{\infty} \sum_{m=-n}^{n}\left\{B_{m n}^{\mu v}(l, j) \mathbf{M}_{m n}^{(3)}(j)+A_{m n}^{\mu v}(l, j) \mathbf{N}_{m n}^{(3)}(j)\right\}\right]
\end{array}
$$

\section{Numerical Results}

This paper introduces the numerical results of multiple scattering by two PEC spheres. The validity of the present mathematical formalism has been investigated by multiparadigm programming language (Matlab) and simulation software (Comsol Multiphysics 5.4). The proposed method efficiency has been analyzed by comparison of Matlab and Comsol numerical results. This article is dedicated to a scattering theory using translation AT for spherical harmonic functions. Specifically, the calculation of the translation coefficients for two PEC spheres and the numerical outcomes of the scattered electric field between two PEC spheres in the near zone region are discussed. Therefore, Equation (48) was used for numerical simulation using a Matlab code. 


\subsection{Validation Test for Two PEC Spheres at Zero Translation Distance}

In the first numerical test, the translation distance between two PEC spheres is zero. This means that the problem of two PEC spheres is reduced into a problem with a single PEC sphere. Then, the single PEC sphere present in a free space is investigated to demonstrate the validity of numerical simulations. For a single PEC sphere, the scattering coefficients $e_{\mu \nu}$ and $f_{\mu \nu}$ can be obtained by using the solution of two linear equations. As a result of the above calculations $g_{\mu \nu}=0$ and $h_{\mu v}=0$, and the use of Equations (42) and (43), the scattered coefficients can be achieved:

$$
\begin{aligned}
e_{\mu v} & =X_{n}(a) a_{\mu v} \\
f_{\mu v} & =Y_{n}(a) b_{\mu v}
\end{aligned}
$$

It occurs when the origin of the $l_{t h}$ and $j_{t h}$ coordinates system are overlapped (i.e., no translation is involved). $m=\mu, n=v, A_{m n}^{\mu \nu} \equiv 1, B_{m n}^{\mu v} \equiv 0$, and $m \neq \mu, n \neq v, A_{m n}^{\mu \nu} \equiv 0$, and $B_{m n}^{\mu v} \equiv 0$. From the previous conditions, the following expressions are obtained $\mathbf{M}_{\mu \nu}^{(3)}(l) \equiv \mathbf{M}_{\mu v}^{(3)}(j), \mathbf{N}_{\mu v}^{(3)}(l) \equiv \mathbf{N}_{\mu v}^{(3)}(j)$. Consequently, the scattered field by using the above Equations (49) and (50) may be written as:

$$
\mathbf{E}_{s}(l)=\sum_{v=1}^{\infty} \sum_{\mu=-v}^{v}\left[e_{\mu v} \mathbf{M}_{\mu v}^{(3)}(l)+f_{\mu v} \mathbf{N}_{\mu \nu}^{(3)}(l)\right]
$$

For this purpose, the following parameters are selected: radius of the sphere $a=0.1 \mathrm{~m}$, frequency $f=0.3 \mathrm{GHz}$, angles of incident $\theta_{i}=0 \mathrm{rad}$, and $\phi_{i}=1 \mathrm{rad}$. Considering the position of cartesian coordinates system with center $x_{r}=y_{r}=z_{r}=0$ and center of the sphere $x_{q}=0, y_{q}=0, z_{q}=0$, the field was measured along the line segment with double of length for the radius of the sphere lying parallel along $x$-axis, which is placed at hight $z=0.3 \mathrm{~m}, y=0$. The numerical results (Matlab code) are comparable with the simulation results (COMSOL Multiphysics 5.4) as shown in Figure 2.
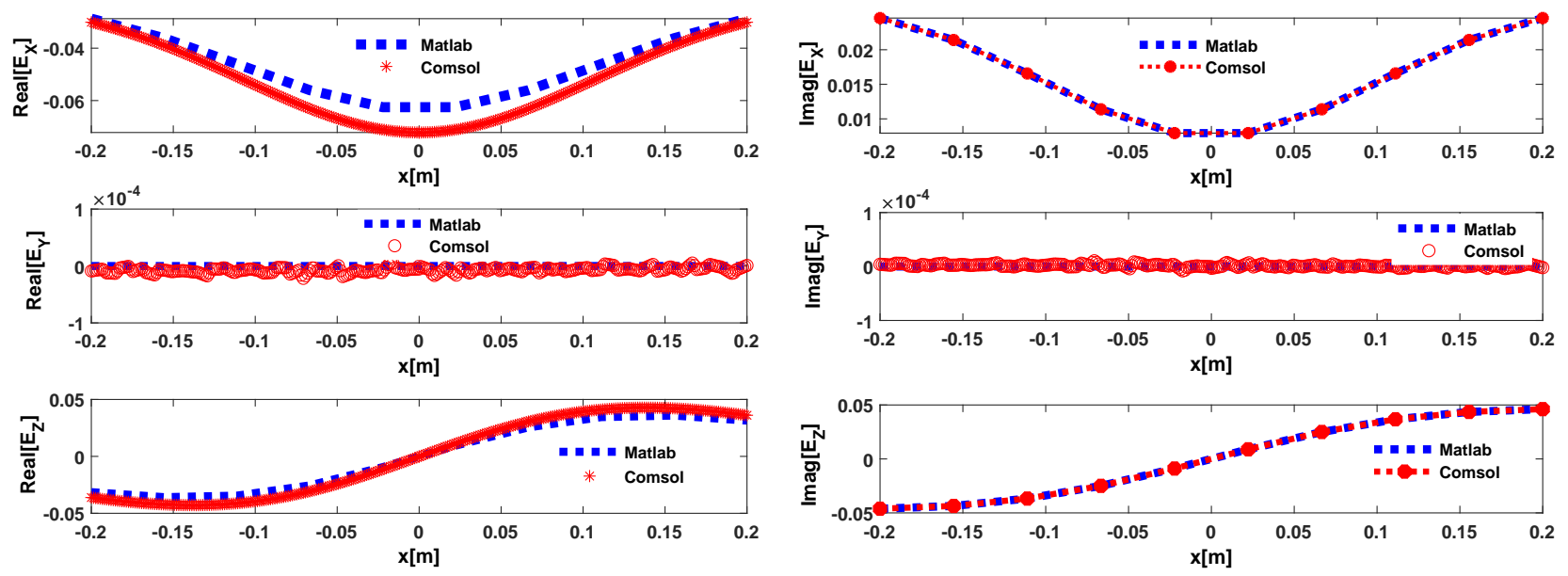

Figure 2. Real part (left) and imaginary part (right) of the scattered electric field by a PEC sphere present in free space. Matlab results (blue color dot lines) are super-imposed on Comsol results (red marker color lines). Here, selected parameters are frequency $f=0.3 \mathrm{GHz}$, radius $a=c=0.1 \mathrm{~m}$.

Further, the scattering behavior with translation of both PEC spheres at different translation distance was investigated. The plots for the scattering electric field are analyzed at three different range of frequencies: $3 \mathrm{MHz}, 0.3 \mathrm{GHz}$, and $3 \mathrm{GHz}$, respectively. The derived mathematical formalism reveals the response of the truncation error for translation AT of spherical harmonics. It is observed that for lower frequency, the numerical results of the Matlab code presents the optimized convergence at lower truncation sum $N$, whereas 
the higher the frequency, the higher the value of truncation sum $N$ for the numerical results of the Matlab code.

\subsection{Case 1: $f=3 \mathrm{MHz}$}

In this section, the numerical results of scattered electric fields have been investigated at a frequency $f=3 \mathrm{MHz}$. Let suppose that the PEC spheres I, II were translated along $z$-axis at $z=0.3 \mathrm{~m}$ and $z=-0.3 \mathrm{~m}$, respectively. The position of the field measuring line can be defined as: the line segment to double the radius of sphere that lies parallel along $x$-axis; that is placed at height $z=0.1 \mathrm{~m}, y=0$. For the analysis of the scattering field pattern, the following parameters have been chosen: radius of spheres I, II $a=c=0.1 \mathrm{~m}$, angles of incident $\theta_{i}=0 \mathrm{rad}$, and $\phi_{i}=1 \mathrm{rad}$. The best comparison between Matlab code and Comsol simulation results are illustrated in Figure 3. The numerical results show that the real part of scattering fields for $E_{x}$ and $E_{z}$ components has a larger amplitude compared to the imaginary part of the same $E_{x}$ and $E_{z}$. The component of electric field $E_{y}$ is equal to zero for both real and imaginary cases.
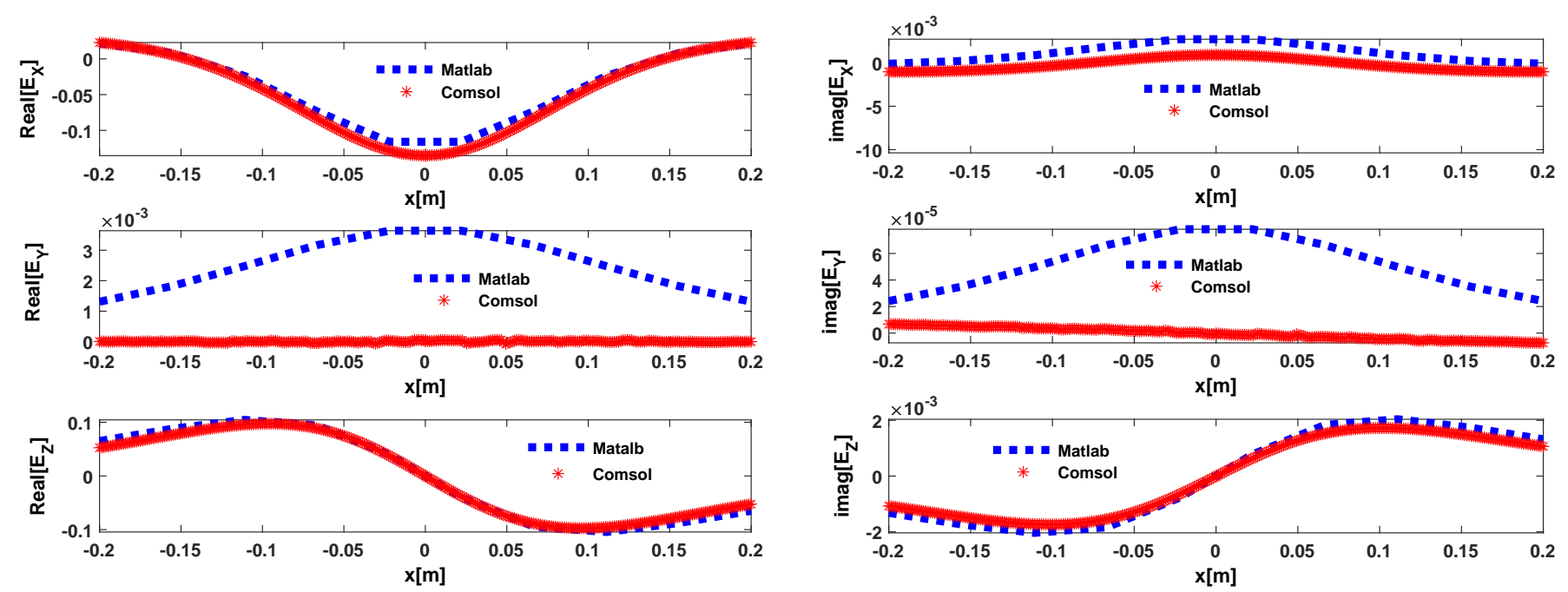

Figure 3. Real part (left) and imaginary part (right) of the scattered electric field by two PEC spheres. In particular, Matlab results (blue color dot lines) are perfectly superimposed on Comsol results (red marker color lines). Sphere I and sphere II are translated at $0.3 \mathrm{~m}$ and $-0.3 \mathrm{~m}$ along $z$-axis, and the selected frequency is $f=3 \mathrm{MHz}$.

Figure 4 presents the translation of both PEC spheres I, II translated along $z$-axis at $z=0.5 \mathrm{~m}$ and $z=-0.5 \mathrm{~m}$. The position of the field measuring line is translated at hight $z=0.2 \mathrm{~m}$. The observed numerical results at a lower values of truncation sum at $N=5$ Matlab code show the perfect matching with Comsol results.

\subsection{Case 2: $f=0.3 \mathrm{GHz}$}

The numerical results of scattered electric fields have been inspected at a frequency $f=0.3 \mathrm{GHz}$. The PEC spheres I, II have translated along $z$-axis at $z=0.5 \mathrm{~m}$ and $z=-0.5 \mathrm{~m}$. The position of the field measuring line is translated along $z$-axis at $z=0.3 \mathrm{~m}$. During our numerical analysis, the chosen parameters were: radius of spheres I, II $a=c=0.1 \mathrm{~m}$, angles of incident $\theta_{i}=0 \mathrm{rad}$, and $\phi_{i}=1 \mathrm{rad}$. The best comparison between Matlab code and Comsol simulation results are illustrated in Figure 5. 

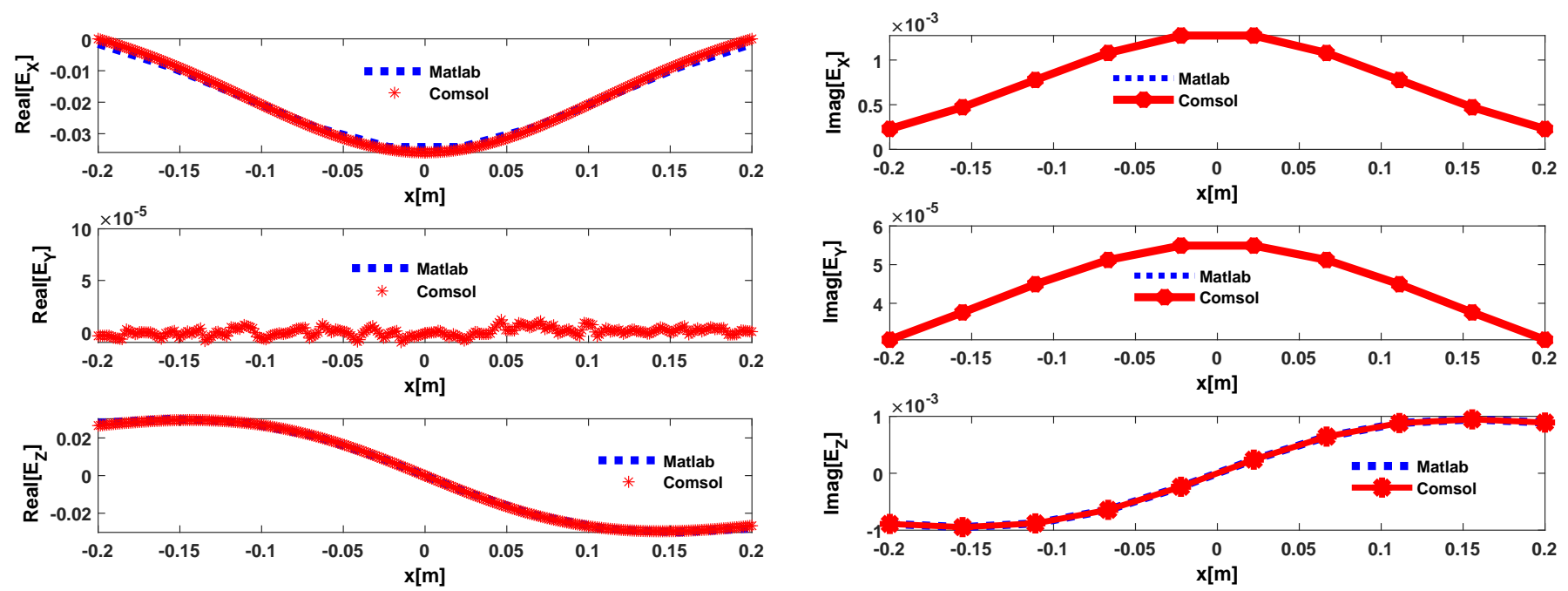

Figure 4. Real part (left) and imaginary part (right) of the scattered electric field by two PEC spheres. In particular, Matlab results (blue color dot lines) are perfectly super-imposed on Comsol results (red marker color lines). Sphere I and sphere II are translated at $0.5 \mathrm{~m}$ and $-0.5 \mathrm{~m}$ along $z$-axis, and the selected frequency is $f=3 \mathrm{MHz}$.
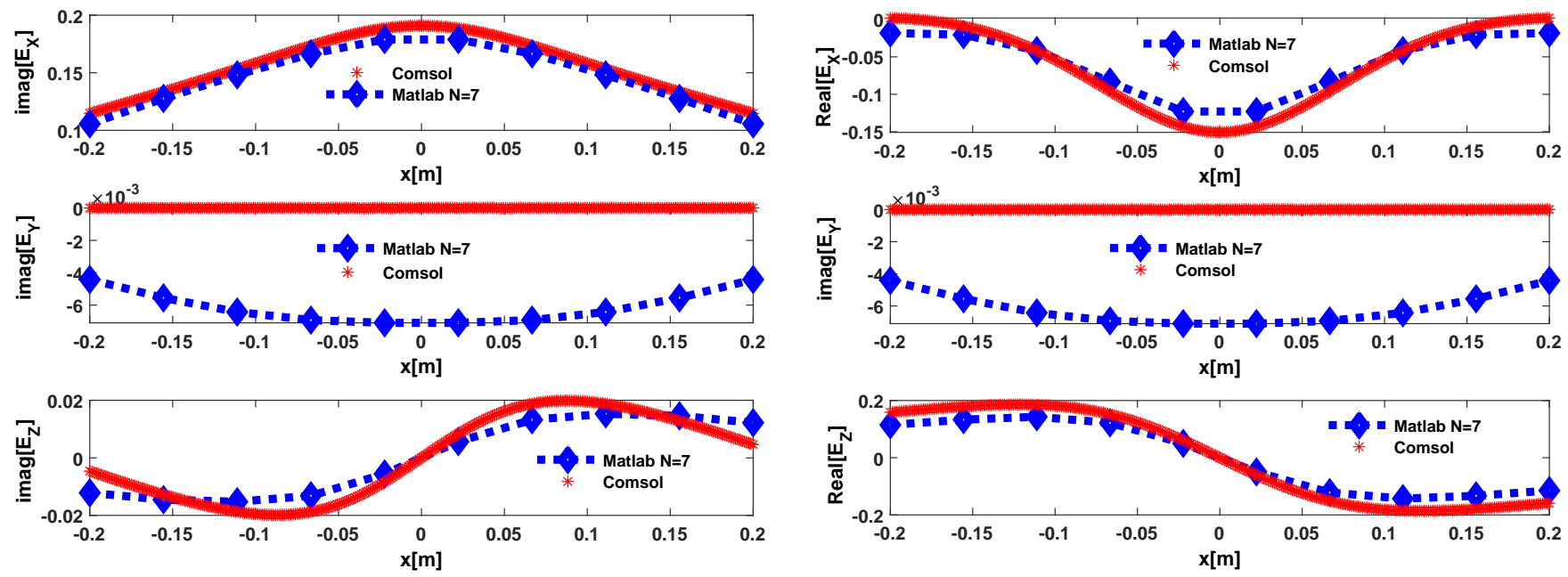

Figure 5. Real part (left) and imaginary part (right) of the scattered electric field by two PEC spheres. In particular, Matlab results (blue color dot lines) are perfectly super-imposed on Comsol results (red marker color lines). Sphere I and sphere II are translated at $0.5 \mathrm{~m}$ and $-0.5 \mathrm{~m}$ along $z$-axis, and the selected frequency is $f=0.3 \mathrm{GHz}$.

The numerical results show that the real part of scattering fields for $E_{z}$ components has a higher amplitude compared to the imaginary part of the same $E_{z}$, while the real part of scattering fields for $E_{x}$ components has a smaller amplitude compared to the imaginary part of the same $E_{x}$. The component of electric fields $E_{y}$ is equal to zero for both real and imaginary cases.

Figure 6 demonstrates the translation of both PEC spheres I, II along $z$-axis at $z=0.4 \mathrm{~m}$ and $z=-0.4 \mathrm{~m}$. The position of the field measuring line is translated at a height $z=0.1 \mathrm{~m}$. The numerical results, for a lower value of truncation sum $N=4$ Matlab code, show fluctuations beyond the results obtained through Comsol. Increasing the truncation sum to $N=37$ Matlab code makes the numerical results perfectly match with Comsol. 

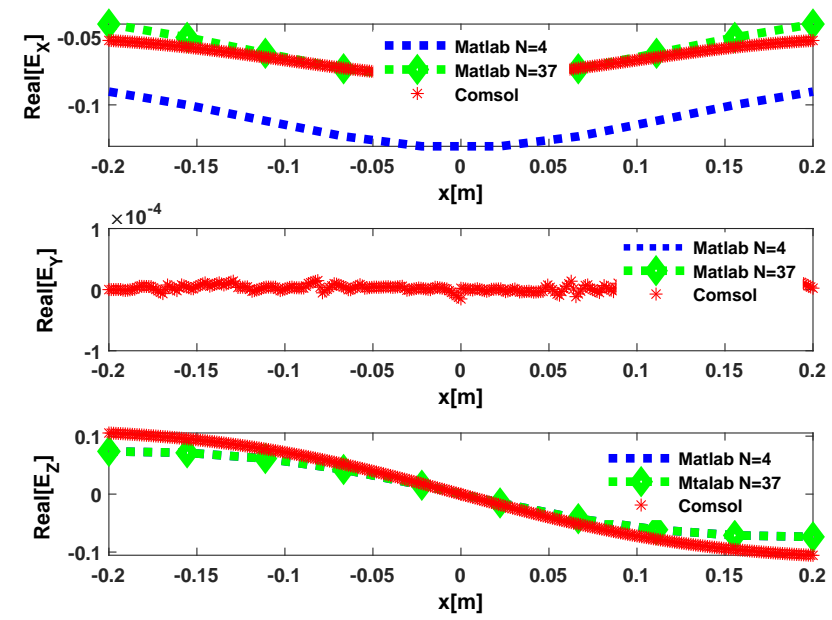
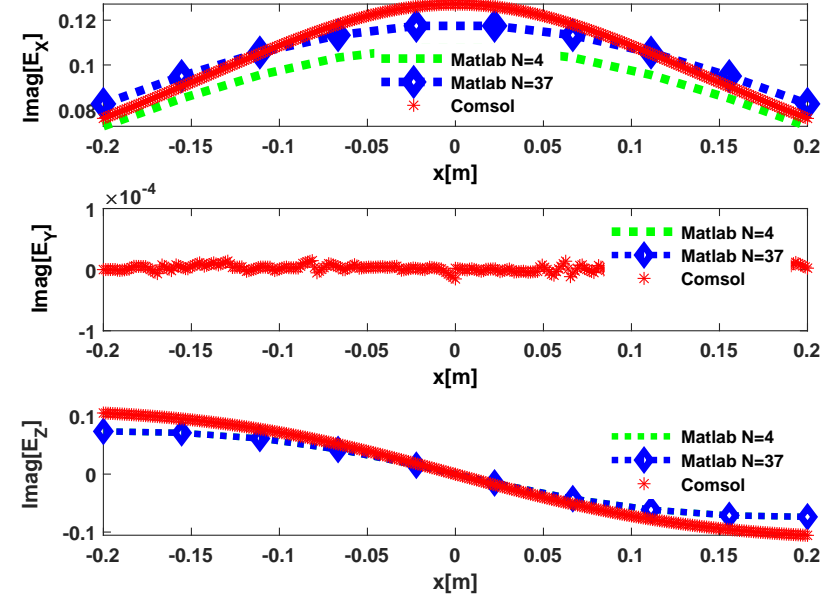

Figure 6. Real part (left) and imaginary part (right) of the scattered electric field by two PEC spheres. The red color lines (Comsol results), blue and green lines (Matlab results) at different value of the truncation sum $N$, sphere I, and sphere II are translated at $0.4 \mathrm{~m}$ and $-0.4 \mathrm{~m}$ along $z$-axis, and the selected frequency is $f=0.3 \mathrm{GHz}$.

\subsection{Case 3: $f=3 \mathrm{GHz}$}

The PEC spheres I, II were translated along $z$-axis at $z=0.08 \mathrm{~m}$ and $z=-0.08 \mathrm{~m}$. The position of the field measuring line is translated along $z$-axis at $z=0.3 \mathrm{~m}$. During our numerical test, the following parameters have been chosen: radius of the spheres I, II $a=c=0.05 \mathrm{~m}$, angles of incident $\theta_{i}=0 \mathrm{rad}, \phi_{i}=1 \mathrm{rad}$, and $f=3 \mathrm{GHz}$. Figure 7 illustrates the numerical results for a lower value of truncation sum $N=5$ Matlab code, showing fluctuations across the Comsol results. Increasing the truncation sum to $N=40$ in Matlab code makes the numerical results perfectly match with Comsol outcomes.
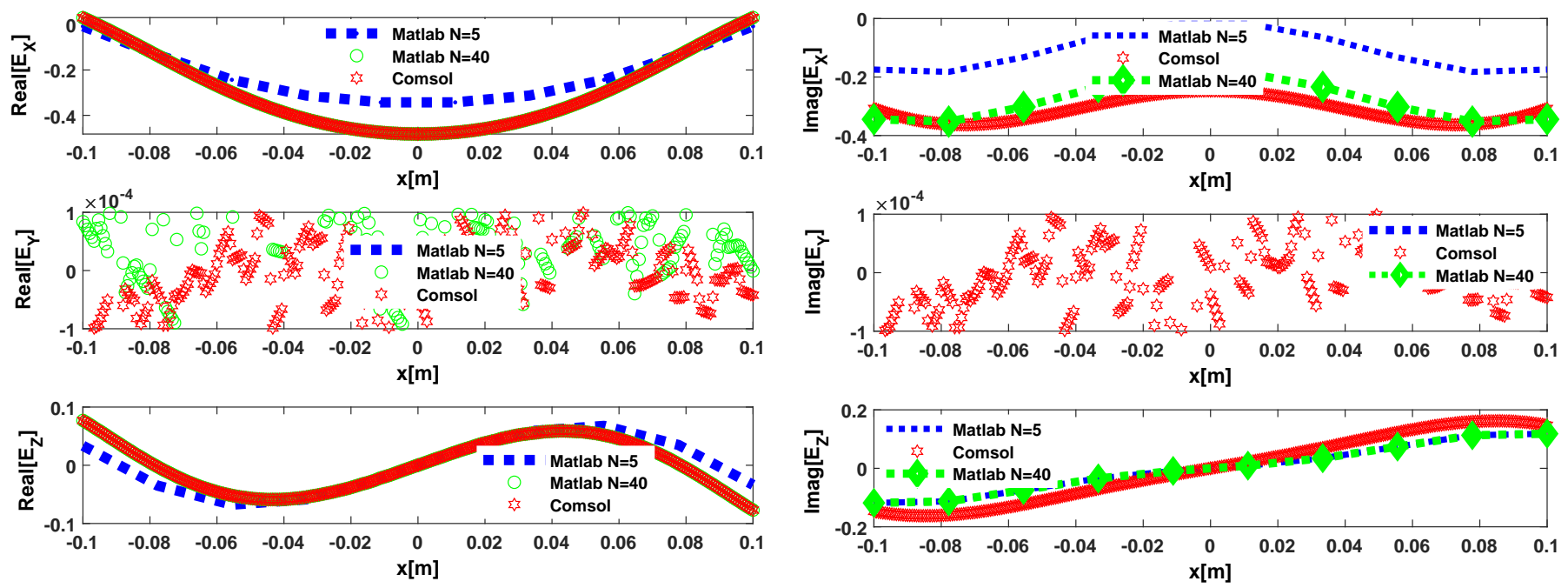

Figure 7. Real part (left) and imaginary part (right) of the scattered electric field by two PEC spheres. The red color lines (Comsol results), blue and green lines (Matlab results) at different value of the truncation sum $N$, sphere I and sphere II are translated at $0.08 \mathrm{~m}$ and $-0.08 \mathrm{~m}$ along $z$-axis, and the selected frequency is $f=3 \mathrm{GHz}$. 


\section{Truncation Error Analysis of Scattered Electric Field Using Vector Translation AT}

The criteria of truncating the inherent infinite series with a finite version of the same to achieve convergence was developed. This leads to a little fluctuation compared to the test of the scattered electric field by two PEC spheres using Comsol and Matlab simulation software, as mentioned in Section 3. The error rate for the proposed approach due to the value of truncation sum $\mathrm{N}$ is investigated in this section. Using Equation (49), the error function for scattered electric field is defined as follows:

$$
\text { err }=\frac{\left\|E_{s}^{n+1}-E_{s}^{n}\right\|}{\left\|E_{s}^{n}\right\|} \quad n=1,2,3 \ldots
$$

where

$$
\left\|E_{s}^{n}\right\|=\sqrt{\sum_{n=1}^{N}\left|E_{s}^{n}\right|^{2}}
$$

The higher value of truncation number $\mathrm{N}$ in the scattered electric field can be employed to tune the accuracy of the solution. Additionally, as the lower values of truncation sum $N$ the error level increases with the variation of frequency. When we increase the number of terms to $N \geq 40$ by using above input, the convergence results are depicted in Figure 8 . Finally, the criteria of truncation behavior related to translation AT reliant directly on frequency was discovered. For higher frequency, the truncation sum $N$ converges at a higher numerical value for translation AT shown in Table 1. This methodology is useful even for spheres as small $r<<\lambda$ and spheres as large $r>>\lambda$. The former solution is very general and applicable to multiple PEC spheres. When sphere I is moving away from sphere II, for both cases real and imaginary numerical results, a higher concentration of scattering electric field pattern is shown. The improvement of our approach will provide a new concept to researchers for developing the deep analysis of the light scattering problem across general distributions of spheres.
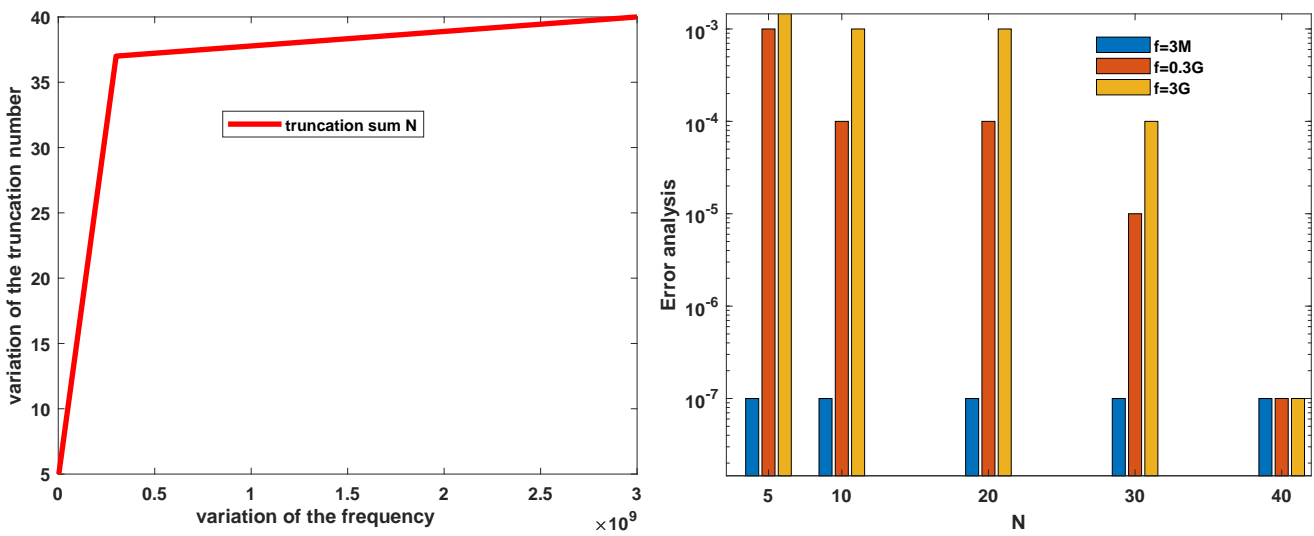

Figure 8. Representation of the truncation variation sum $N$ (left) and its convergence solution with the variation of different frequency $f=3 \mathrm{M}, f=0.3 \mathrm{G}, f=3 \mathrm{G}$ (right).

Table 1. Variation of truncation number $\mathrm{N}$ with respect to frequency.

\begin{tabular}{cccc}
\hline$\#$ & Frequency & Size of a Sphere & $\begin{array}{c}\text { Convergence of the Solution } \\
\text { (Truncation Number N) }\end{array}$ \\
\hline 1 & $3 \mathrm{MHz}$ & $0.1 \mathrm{~m}$ & $\mathrm{~N}=5$ \\
\hline 1 & $0.3 \mathrm{GHz}$ & $0.1 \mathrm{~m}$ & $\mathrm{~N}=37$ \\
\hline 2 & $3 \mathrm{GHz}$ & $0.05 \mathrm{~m}$ & $\mathrm{~N}=40$ \\
\hline
\end{tabular}




\section{Conclusions}

This paper presents and explores multiple scattering by two PEC spheres through computer simulations. A method of translation AT for spherical vector wave equation was introduced. To describe this problem, a Cruzan's formalism for vector translation coefficients was proposed. The scattering electric field by two PEC spheres in a near zone region was calculated. The translation of spheres I, II along the $z$-axis was thought out and resolved to understand the scattering behavior. The results of our calculations were investigated using a numerical approach. The numerical results were compared with the Comsol simulation models and both software results were found to be perfectly matched one another, which gives a fairly good validation.

In summary, the work presented in this letter has investigated the finite truncation sum $N$. The convergence of the solution is useful to determine the scattering efficiency of a homogeneous spherical scatterer by a plane wave. From a practical point of view, the proposed mathematical expressions are clearly useful to facilitate fast simulations of light scattering phenomena. It should be noted that this truncation criterion applies to homogeneous spheres, whereas other shapes result in their own scattering coefficients.

We aim to build on shared fundamentals, highlight the most pressing research challenges, and exchange state-of-the-art methodologies and approaches. This all-community approach will promote solutions to those issues facing many practical applications in complex media through the rigorous underpinning of mathematical techniques and the development of effective computational methods.

Author Contributions: The authors S.B. conceived the presented idea. S.B. developed the formulation or evolution of overarching research goals and aims. and performed the computations. Following co-authors (M.N., L.D., F.M., F.F.) encouraged first author to investigate a specific aspect and supervised the findings of this work. All authors provided critical feedback and helped in equal contribution to the formation of the research article. All authors have read and agreed to the published version of the manuscript.

Funding: This research received external funding from Sapienza university of Rome Italy.

Data Availability Statement: The authors confirm that the data supporting the findings of this study are available within the article.

Conflicts of Interest: The authors declare no conflict of interest.

\section{References}

1. Oguchi, T. Electromagnetic wave propagation and scattering in rain and other hydrometeor. Proc. IEEE 1983, 71, 1029-1078. [CrossRef]

2. Batool, S.; Dinia, L.; Frezza, F.; Mangini, F.; Nisar, M. Electromagnetic interaction with a monodispersed system in sedimentation equilibrium. In Proceedings of the 2020 XXXIIIrd General Assembly and Scientific Symposium of the International Union of Radio Science, Rome, Italy, 29 August-5 September 2020; pp. 1-4.

3. Borghese, F.; Denti, P.; Saija, R.; Toscano, G.; Sindoni, O.I. Use of group theory for the description of electromagnetic scattering from molecular systems. J. Opt. Soc. Am. A 1984, 2, 183-191. [CrossRef]

4. Mangini, F.; Dinia, L.; Frezza, F. Electromagnetic scattering by a cylinder in a lossy medium of an inhomogeneous elliptically polarized plane wave. J. Telecommun. Inf. Technol. 2019, 4, 36-42. [CrossRef]

5. Dinia, L.; Mangini, F.; Frezza, F. Electromagnetic scattering of inhomogeneous plane wave by ensemble of cylinders. J. Telecommun. Inf. Technol. 2020, 3, 86-92. [CrossRef]

6. Mangini, F.; Tedeschi, N. Scattering of an electromagnetic plane wave by a sphere embedded in a cylinder. J. Opt. Soc. Am. A 2017, 34, 760-769. [CrossRef]

7. Batool, S.; Naqvi, Q.A.; Fiaz, M.A. Scattering from a cylindrical obstacle deeply buried beneath a planar non-integer dimensional dielectric slab using Kobayashi potential method. Opt.-Int. J. Light Electron. Opt. 2018, 153, 95-108. [CrossRef]

8. Batool, S.; Nisar, M.; Mangini, F.; Frezza, F.; Fazio, E. Polarization Imaging for Identifying the Microscopical Orientation of Biological Structures. In Proceedings of the URSI GASS Conference, Rome, Italy, 29 August-5 September 2020.

9. Fazio, E.; Batool, S.; Nisar, M.; Mangini, F.; Frezza, F. Recognition of bio-structural anisotropy by polarization-resolved imaging. Sensor 2021, submitted.

10. Batool, S.; Nisar, M.; Mangini, F.; Frezza, F.; Fazio, E. Scattering of Light from the Systemic Circulatory System. Diagnostics 2020, 10, 1026. [CrossRef] [PubMed] 
11. Batool, S.; Frezza, F.; Mangini, F.; Simeoni, P. Introduction of Radar Scattering Application in Remote Sensing and Diagnostics. Atmosphere 2020, 11, 517. [CrossRef]

12. Gastellu, E.J.P.; Demarez, V.; Pinel, V.; Zagolski, F. Modeling radiative transfer in heterogeneous 3-D vegetation canopies. Remote Sens. Environ. 1996, 58, 131-156. [CrossRef]

13. Ioannidou, M.P.; Skaropoulos, N.C. Study of interactive scattering by clusters of spheres. JOSA A 1995, 12, 1782-1789. [CrossRef]

14. Liang, C.; Lo, Y.T. Scattering by two spheres. Radio Sci. 1967, 12, 1481-1495. [CrossRef]

15. Olaofe, G.O. Scattering by two cylinders. Radio Sci. 1970, 5, 351-360. [CrossRef]

16. Fuller, K.A. Optical resonances and two-sphere systems. Appl. Opt. 1991, 30, 4716-4731. [CrossRef]

17. Bruning, J.; Lo, Y. Multiple scattering of EM waves by spheres part I Multipole expansion and ray-optical solutions. IEEE Trans. Antennas Propag. 1971, 19, 378-390. [CrossRef]

18. Wang, Y.M.; Chew, W.C. A recursive T-matrix approach for the solution of electromagnetic scattering by many spheres. IEEE Trans. Antennas Propag. 1993, 41, 1633-1690. [CrossRef]

19. Friedman, B.; Russek, J. Addition theorems for spherical waves. Q. Appl. Math. 1954, 1, 13-23. [CrossRef]

20. Stein, S. Addition theorems for spherical wave functions. Q. Appl. Math. 1961, 19, 15-24. [CrossRef]

21. Cruzan, O.R. Translational addition theorems for spherical vector wave functions. Q. Appl. Math. 1962, 20, 33-40. [CrossRef]

22. Mackowski, D.W. Analysis of radiative scattering for multiple sphere configurations. Proc. R. Soc. London. Ser. A Math. Phys. Sci. 1991, 433, 599-614.

23. Xu, Y.L. Electromagnetic scattering by an aggregate of spheres. Appl. Opt. 1995, 34, 4573-4588. [CrossRef]

24. Xu, Y.L. Fast evaluation of the Gaunt coefficients. Math. Comput. 1996, 65, 1601-1612. [CrossRef]

25. Xu, Y.L. Efficient evaluation of vector translation coefficients in multiparticle light-scattering theories. J. Comput. Phys. 1998, 139, 137-165. [CrossRef]

26. Batool, S.; Frezza, F.; Mangini, F.; Xu, Y.L. Scattering from multiple PEC sphere using translation addition theorems for spherical vector wave function. J. Quant. Spectrosc. Radiat. Transf. 2020, 248, 106905. [CrossRef]

27. Batool, S.; Benodetti, A.; Frezza, F.; Mangini, F.; Xu, Y.L. Effect of finite terms on the truncation error of addition theorems for spherical vector wave function. In Proceedings of the Photonics and Electromagnetics Research Symposium-Spring (PIERSSpring), Rome, Italy, 17-20 June 2019; pp. 2795-2801.

28. Neves, A.A.; Pisignano, D. Effect of finite terms on the truncation error of Mie series. J. Comput. Phys. 2012, 37, $2418-2420$.

29. Wiscombe, W.J. Improved Mie scattering algorithms. Appl. Opt. 1980, 19, 1505-1509. [CrossRef]

30. Frezza, F.; Mangini, F.; Tedeschi, N. Introduction to electromagnetic scattering: Tutorial. J. Opt. Soc. Am. 2018, 35, 163-173. [CrossRef] [PubMed]

31. Frezza, F.; Mangini, F.; Tedeschi, N. Introduction to electromagnetic scattering, part II: Tutorial. J. Opt. Soc. Am. 2020, 37, 1300-1315. [CrossRef]

32. Mackowski, D.W. Calculation of total cross sections of multiple-sphere clusters. J. Opt. Soc. Am. A 1994, 11, 2851-2861. [CrossRef] 